\title{
A Nomogram to Predict Lifestyle Factors for Recurrence of Large-Vessel Ischemic Stroke
}

This article was published in the following Dove Press journal:

Risk Management and Healthcare Policy

\author{
Zhi-Xin Huang $\mathbb{D}^{1-3}$ \\ Shumin Yuan ${ }^{4}$ \\ Dongshi $\mathrm{Li}^{1,3}$ \\ Hong $\mathrm{Hao}^{2}$ \\ Zhenguo Liu $^{2}$ \\ Jianguo $\operatorname{Lin}^{1,3}$
}

'Department of Neurology, Guangdong Second Provincial General Hospital, Guangzhou, Guangdong, China; ${ }^{2}$ Center for Precision Medicine and Division of Cardiovascular Medicine, Department of Medicine, University of Missouri School of Medicine, Columbia, MO, USA; ${ }^{3}$ Department of Neurology, The Second School of Clinical Medicine, Southern Medical University, Guangzhou, Guangdong, China; ${ }^{4}$ Department of Biochemistry and Molecular Biology, Guilin Medical University, Guilin, Guangxi, China
Correspondence: Zhi-Xin Huang Department of Neurology, Guangdong Second Provincial General Hospital, 466 Middle Xingang Road, Guangzhou, 510317, Guangdong, China

Tel/Fax +862089168080

Email hzxd6@I63.com

Zhenguo Liu,

Center for Precision Medicine and

Division of Cardiovascular Medicine,

Department of Medicine, University of

Missouri School of Medicine, Columbia, MO, USA

Email liuzheng@health.missouri.edu
Background: Stroke is the leading cause of morbidity and mortality in China. Recurrent stroke (RS) could occur in a significant portion of patients with ischemic stroke with devastating consequence.

Methods: To investigate the association between lifestyle and the risk of RS in Chinese patients with acute large-vessel ischemic stroke (ALVIS). A total of 258 patients with ALVIS were recruited in the study (median age 63 years, $30.6 \%$ female), and followed for a median of 366 days. The primary outcomes were first RS. Cox Regression and Akaike information criterion were used to establish the best-fit nomograms.

Results: During follow-up, 38 of 258 (14.7\%) participants had the primary endpoint event. After adjusting for confounding factors in multivariate Cox regression analysis, healthy lifestyles, including bland diet (hazard ratio [HR], 0.365; 95\% CI, 0.138-0.965), daily fruit consumption (HR, 0.474; 95\% CI, 0.238-0.945), good sleep (HR, 0.364; 95\% CI, 0.180-0.739), housework: HR (0.461; 95\% CI, 0.200-1.065), and HDL (HR, 0.329; 95\% CI, $0.130-0.831)$ were associated with significantly decreased risk for RS after ALVIS, while smoking was associated with a substantial increase in RS risk (HR, 2.590; 95\% CI, 1.340-5.005) and included into the nomogram. A weighted point (from 0 to 100) was given to each risk factor, and the total points could be used to predict the probability of RS for the patient. Conclusion: The nomogram shows that healthy lifestyles (bland diet, daily fruit consumption, good sleep, cigarette cessation, and housework) were important for reducing RS in patients with ALVIS.

Keywords: recurrent event, lifestyle, ischemic stroke, stroke, nomogram

\section{Introduction}

Acute ischemic stroke (AIS) is the leading cause of adult morbidity and mortality in developing countries. ${ }^{1}$ Patients with ischemic stroke have a poor prognosis and a high risk of recurrent stroke (RS). ${ }^{2,3}$ RS was defined as a new neurologic deficit lasting at least 24 hours after the initial stroke, without signs of increased intracranial pressure or hemorrhagic transformation, was associated with a new ischemic lesion on brain imaging. ${ }^{4}$ Compared with other stroke subtypes, acute large-vessel ischemic stroke (ALVIS) is associated with a high risk of RS despite optimal medical treatment. ${ }^{5,6}$ Clinically, patients with RS usually suffer more neurological damages, and more challenging to treatment, thus a higher mortality rate than those with only the initial event. Therefore, it is critically important to take proactive measures to prevent RS for patients with ALVIS in addition to standard treatments for optimal outcomes.

Over $90 \%$ of ischemic strokes could be associated with modifiable risk factors and poor lifestyles. ${ }^{7,8}$ Epidemiological studies have shown that a variety of factors 
are associated with RS, including hypertension, diabetes, hyperlipidemia, and coronary heart disease, after the initial ischemic stroke. However, the impact of physical activity and lifestyle modification on secondary prevention of stroke has not been well studied in the stroke prevention trial, although lifestyle modification has been documented to be associated with decreased risk of stroke as the initial event in patients with cerebral atherosclerosis. ${ }^{9}$ Lifestyle modifications could be easily achieved without adverse effects or additional cost. Among the recommended lifestyle modifications, healthy diet, losing weight, quitting smoking, and regular physical activity are the most common and well-received items. ${ }^{10}$

However, there is very limited data on the impact of lifestyle modifications on reducing the risk of RS after ALVIS. It is known that ALVIS is an important cause of ischemic stroke with significant brain damages, high recurrence rate, and poor outcome, which is usually worse than other forms of stroke. ${ }^{11-14}$ Recently, we reported that lifestyle modification could significantly reduce the risk of RS for patients with all forms of ischemic stroke. It is known that stroke is not one pathological condition, but a highly heterogeneous disorder. The most important etiologies of ischemic stroke are larger-artery atherosclerosis (LAA), intracranial small-artery disease, and cardioembolism. Less common causes of stroke include cerebral vasculitis, coagulopathies, and hematologic disorders. Still, a significant portion of ischemic stroke cases remains etiologically undefined. Different subtypes of ischemic stroke may have different risk factors, clinical features, and prognoses. Our previous study was designed to investigate the effect of lifestyle factors on risk reduction for patients with all forms of acute ischemic stroke. The present study was to focus on defining the effect of lifestyle modification on RS risk for the patients with ALVIS since these patients are associated with a much higher risk of RS.

Lifestyle is very diverse and complex, and everyone has a different lifestyle with a wide range of variables. Nomograms are visualized graphical representations of statistical prediction models to calculate the numerical probability of an endpoint event. These models have been successfully applied to the studies on malignant tumors. ${ }^{15,16}$ Using the established prognostic variables, nomograms are able to generate individualized predictive values that could be used to optimize patient management including counseling and risk stratification. To our knowledge, predictive nomograms for the evaluation on RS risk in patients with ALVIS have not been well established. In the present study, we developed a simple and clinically relevant nomogram to predict the relationship between lifestyle and RS in patients with ALVIS.

\section{Methods}

All procedures performed in the present study in human participants were in accordance with the 1964 Helsinki declaration and its later amendments, and in line with the local and national ethical standards. The study protocol was reviewed and approved by the Clinical Research Ethics Committee of the Guangdong Second Provincial General Hospital, Guangzhou, Guangdong, China. Written informed consent was obtained from all subjects prior to their participation.

\section{Patient Population}

All consecutive patients with first AIS (within 7 days of symptom onset) who were hospitalized from November 2012 to January 2014 at the Guangdong Second Provincial General Hospital, Guangzhou, Guangdong, China, a large teaching hospital that provides comprehensive cares including neurological care to more than 1 million population in Southern China, were recruited to this study. The classification of AIS was determined based on the criteria of Trial of Org 10,172 in Acute Stroke Treatment (TOAST) as described. ${ }^{17}$ Only the patients with ALVIS were included in the present study. The exclusion criteria were as follows: 1) cerebral hemorrhage confirmed by computed tomography (CT) or magnetic resonance imaging (MRI); 2) within thrombolytic time window (within 4.5 h); 3) significant liver, heart, or kidney failure; 4) any form of malignance; 5) previous ischemic stroke prior to the current hospitalization; 6) severe mental illness or dementia; 7) history of carotid stenting or carotid endarterectomy; 8) Lacunar cerebral infarction; and 9) cerebral infarction with probable cardiac source of embolism (Figure 1). Since ischemic stroke patients with severe initial disability have a tendency to visit hospitals early with poor long-term outcome that could prevent follow up, ${ }^{18-23}$ patients with severe initial disability were also excluded from the study.

\section{Clinical Assessment}

All patients underwent neurological function examination, biochemical tests, electrocardiogram, cervical vascular color ultrasound, cardiac color ultrasound, and brain and carotid artery MR imaging examination. The neurological status for the patients at admission was determined using the National Institute of Health Stroke Scale (NIHSS; higher scores indicate greater severity). Peripheral blood samples were collected 


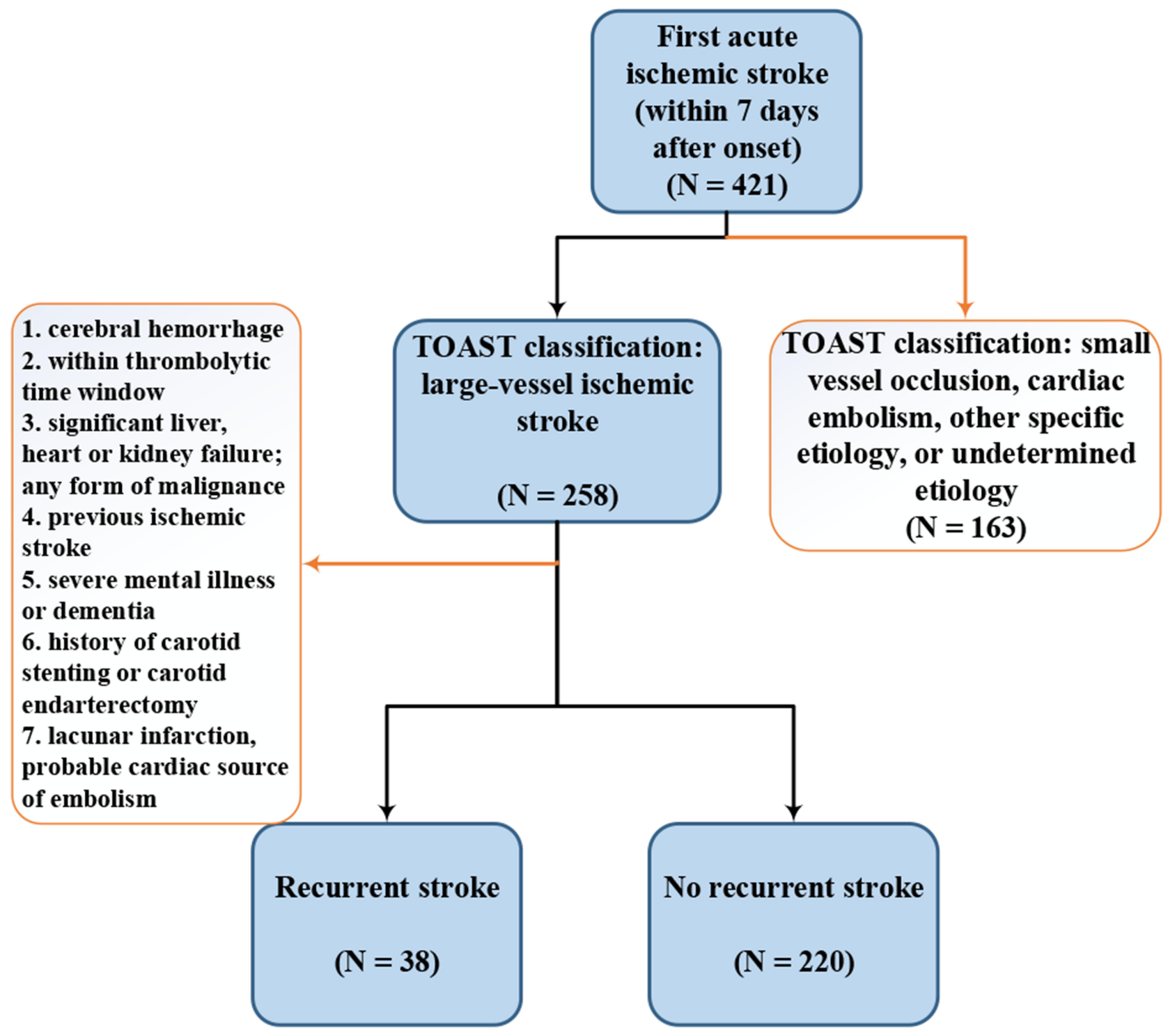

Figure I Flow chart for the patient-selection criteria.

from each subject after approximately 10 hours of fasting for biochemical analysis. In addition, the diagnostic imaging examinations were completed within 72 hours of admission. Patient data, including demographic data, stroke risk factors (such as hyperlipidemia, diabetes, hypertension, and history of ischemic heart disease), and information on medications, lifestyle, and dietary preferences were collected from medical records and during follow-up visits. ${ }^{24}$ Body Mass Index (BMI) was calculated by height and weight assessment. Fasting blood pressure was measured in the morning after waking up, the average of the three measurements with the interval of $2 \mathrm{~min}$ between two blood pressure measurements was used for analysis. Blood pressure grading was as: 0 point for $<140 \mathrm{~mm} \mathrm{Hg}$ systolic or $<90 \mathrm{~mm} \mathrm{Hg}$ diastolic; 1 point indicates 140-159 mm Hg systolic or 90-99 mm Hg diastolic; 2 for 160-179 mm Hg systolic or 100-109 mm Hg diastolic; 3 for $\geq 180 \mathrm{~mm} \mathrm{Hg}$ systolic or $\geq 110 \mathrm{~mm} \mathrm{Hg}$ diastolic. Selfreported education level (college or above, secondary, primary, no education) was obtained. The culprit vessels for AIS were divided into anterior circulation, posterior circulation, both anterior circulation and posterior circulation.

\section{Definitions of RS and Lifestyle}

$\mathrm{RS}$ was defined as a new neurologic deficit lasting for at least 24 hours after the initial stroke and was confirmed by CT/MR and could not be attributed to edema, mass effect, or hemorrhagic transformation. Follow-up was conducted by trained interviewers via telephone at 1,3,6, and 12 months, and 
annually thereafter. Data on RS (including recurrent cerebral infarction and cerebral hemorrhage) were obtained from patients and caregivers during the follow-up.

Healthy lifestyle was defined as described in detail previously, ${ }^{25}$ and briefly stated as following: 1) Bland diet: light, not salty, not spicy, non-greasy food. 2) Daily consumption of fresh fruit: consumption of unprocessed fruit, excluding frozen, dried, canned fruit or fruit juice. 3) Good sleep: absence of sleep abnormalities (including taking longer than $30 \mathrm{~min}$ to fall asleep, or frequent interruptions of sleep at night, or earlier wake up than usual) in a few nights per week. ${ }^{26}$ All patients were asked to record their daily sleep pattern and quality during the period of follow-up, included lights-out time, time from light off to sleep (less or more than half hour), number of nighttime awakenings, and number of awakenings after falling asleep, and feeling of freshness in the morning (yes or no). The data on self-reported sleep quality were collected at each follow-up visit for the period from prior visit. 4) Housework: daily household activities (such as house cleaning, dish washing, self-cooking, and ironing) for at least three hours a day for most of the week as described. ${ }^{27}$ 5) Physical activity: aerobic exercise for more than 30 minutes at least twice a week as described. ${ }^{28}$ During the follow-up, information on lifestyle was regularly obtained from patients as well as relatives or caregivers. 5) Smoking was defined as current smokers or quitting smoking within last 5 years. And, 7) drinking: subjects who drank more than five times per week were defined as having a drinking habit.

\section{Statistical Analysis}

The normality of each continuous variable was tested by using Kolmogorov-Smirnov Z-test. Continuous variables were presented as mean \pm standard deviation (SD) or median (interquartile distance). Qualitative variables were expressed as absolute frequencies and percentages. Forward elimination Cox proportional hazards regression models were used to calculate adjusted relative risks for RS at 1 year, in which we included the potential confounding factors associated with $P<0.15$ in the univariate analyses. A Cox regression of the $\log$ hazard ratio on a covariate with a standard deviation of 0.643 based on a sample of 258 observations achieved $81 \%$ power at a 0.05 significance level to detect a regression coefficient equal to -0.75 . A multiple regression of the variable of interest on the other covariates in the Cox regression was expected to have an R-Squared of 0.11 for an anticipated event rate of 0.15 . Participants who did not have the endpoint events were censored on the last visit or at end of the study. Hazard ratios (HR) and 95\% confidence intervals (CI) were calculated for each risk factor, and the nomograms were built with potential risk factors. The performance of the nomogram was assessed by Harrell's concordance index (C-index). Both discrimination and calibration were evaluated on the original study cohort using bootstrapping with 1000 resamples. Statistical analyses were performed with SPSS version 25.0 (IBM, New York, NY, USA) and $\mathrm{R}$ statistical software (R, version 3.5.1; R Project). Twosided $P$ values $<0.05$ were considered statistically significant.

\section{Results}

The demographic data and clinical characteristics of the subjects are shown in Table 1. A total of 258 patients (median age of 63 years, $30.6 \%$ female) with ALVIS were included in the study and followed up for a median of 366 days (interquartile range: $363-367$ days). A total of 38 patients (14.7\%) had the primary endpoint event of RS during 1 year of follow-up. Based on the study sample size of 258 , a statistical power of $81 \%$ was achieved at the significance level of 0.05 . Overall, $94.2 \%$ of patients had $\geq 1$ stroke risk factors including hypertension, dyslipidemia, diabetes, smoking, drinking, and previous ischemic heart disease. The common risk factors were: hypertension $(70.9 \%$ of the study population, 95\% CI 65.1-76.1\%) and dyslipidemia (43.4\%, 95\% CI $37.5-49.5 \%)$, diabetes $(43.0 \%, 95 \%$ CI $37.1-49.1 \%)$, smoking (31.4\%, 95\% CI 26.0-37.3\%), drinking $(7.0 \%, 95 \%$ CI $4.5-10.8 \%)$, and previous ischemic heart disease $(4.7 \%, 95 \%$ CI $2.7-8.0 \%)$. The percentages of subjects with $1,2,3$, and $\geq 4$ risk factors were $26.4 \%$ (95\% CI $21.4-32.1 \%$ ), 35.7\% (95\% CI 30.1-41.7\%), 27.1\% (95\% CI $22.1-32.9 \%$ ), and $5.0 \%$ (95\% CI 3.0-8.4\%), respectively. Although the percentage of women in the present study was relatively low, there was no sex difference between stroke recurrence and non-recurrence groups (Table 1).

\section{Outcome Events by Dietary Preferences}

Univariate cox analysis showed a significant reduction in the rate of RS during follow-up in the patients on a bland diet compared with those on a non-bland diet ( 86.8 versus 96.8 per 100 patient-years, respectively, HR, 0.286; 95\% CI, 0.112-0.734, $P=0.009$; Table 1). Subjects with daily fruit consumption also had significantly lower risk for RS than those without (36.8 per 100 patient-years versus 57.7, respectively, HR, 0.467; 95\% CI, 0.241-0.902; $P$ value 0.023). Multivariate Cox regression analyses demonstrated that a bland diet or daily fruit consumption significantly reduced the risk of RS by $63.5 \%$ and $52.6 \%$, respectively (adjusted HR: $0.365,95 \%$ CI: $0.138-0.965, P=0.042$; 
Table I Comparison of Demographic and Therapeutic Characteristics of the Two Study Groups (Recurrent Stroke or Not)

\begin{tabular}{|c|c|c|c|c|}
\hline \multirow[t]{2}{*}{ Parameters } & \multicolumn{2}{|l|}{ Recurrent Stroke } & \multirow[t]{2}{*}{ Unadjusted HR } & \multirow[t]{2}{*}{$P$ value } \\
\hline & Yes $(n=38[14.7 \%])$ & No $(n=220[85.3 \%])$ & & \\
\hline \multicolumn{5}{|l|}{ Demographics } \\
\hline Female, n (\%) & $10(26.3)$ & $69(31.4)$ & $0.80 I(0.389-1.650)$ & 0.548 \\
\hline Education level, n (\%) & & & $0.858(0.608-1.212)$ & 0.386 \\
\hline College or above & I (2.6) & $13(5.9)$ & & \\
\hline Secondary & $7(18.4)$ & $51(23.2)$ & & \\
\hline Primary & $15(39.5)$ & $70(31.8)$ & & \\
\hline No education & $15(39.5)$ & $86(39.1)$ & & \\
\hline Living alone, n (\%) & I (2.6) & $2(0.9)$ & $2.985(0.409-21.763)$ & 0.281 \\
\hline BMI, median (IQR) & $22.49(21.48-23.88)$ & $23.15(22.03-24.97)$ & $0.874(0.755-1.012)$ & 0.072 \\
\hline \multicolumn{5}{|l|}{ Dietary preferences, n (\%) } \\
\hline Sweet & I (2.6) & $2(0.9)$ & $3.092(0.424-22.544)$ & 0.265 \\
\hline Salty & $5(13.2)$ & $7(3.2)$ & 3.198 (1.248-8.197) & 0.015 \\
\hline Bland & $33(86.8)$ & $213(96.8)$ & $0.286(0.112-0.734)$ & 0.009 \\
\hline Greasy & $0(0)$ & $2(0.9)$ & $0.049(0-364,198.870)$ & 0.709 \\
\hline Daily fruit consumption (yes) & $14(36.8)$ & $127(57.7)$ & $0.467(0.24 I-0.902)$ & 0.023 \\
\hline Age, median (IQR), years & $63(50.5-69.0)$ & $64(55.0-72.0)$ & $0.987(0.96 \mathrm{I}-1.013)$ & 0.317 \\
\hline \multicolumn{5}{|l|}{ Medical history } \\
\hline Hypertension, n (\%) & $28(73.7)$ & $155(70.5)$ & $1.152(0.560-2.372)$ & 0.700 \\
\hline Diabetes mellitus, n (\%) & $19(50.0)$ & $92(4 \mid .8)$ & $1.349(0.7 \mid 4-2.549)$ & 0.356 \\
\hline History of dyslipidemia, n (\%) & $16(42.1)$ & $96(43.6)$ & $0.928(0.487-1.767)$ & 0.820 \\
\hline IHD, n (\%) & $4(10.5)$ & $8(3.6)$ & $2.544(0.903-7.172)$ & 0.077 \\
\hline Smoking, n (\%) & $18(47.4)$ & $63(28.6)$ & $2.03 \mathrm{I}(\mathrm{I} .075-3.84 \mathrm{I})$ & 0.029 \\
\hline Drinking, n (\%) & $5(13.2)$ & $13(5.9)$ & $2.090(0.816-5.356)$ & 0.125 \\
\hline \multicolumn{5}{|l|}{ Medications } \\
\hline Antiplatelet, n (\%) & $34(89.5)$ & $194(88.2)$ & I. $148(0.407-3.236)$ & 0.794 \\
\hline Antihypertensive, n (\%) & 27 (7I.1\%) & $148(67.3)$ & I.I 179 (0.585-2.377) & 0.645 \\
\hline Cholesterol-Reducer, n (\%) & $35(92.1)$ & $193(87.7)$ & $1.582(0.486-5.142)$ & 0.446 \\
\hline Diabetic medication, n (\%) & $17(44.7)$ & $86(39.1)$ & $1.233(0.650-2.337)$ & 0.521 \\
\hline \multicolumn{5}{|l|}{ Laboratory measures } \\
\hline Hemoglobin AIC, mmol/L & $6.60(5.68-8.60)$ & $6.20(5.80-7.50)$ & $1.154(0.99 \mid-1.344)$ & 0.065 \\
\hline TG, median (IQR), mmol/L & $1.55(1.08-2.00)$ & $1.40(1.08-2.00)$ & $0.980(0.747-1.284)$ & 0.882 \\
\hline TC, median (IQR), mmol/L & $4.23 \pm 1.13$ & $4.76 \pm 1.42$ & $0.773(0.608-0.984)$ & 0.037 \\
\hline $\mathrm{HDL}$, median (IQR), mmol/L & $0.90(0.80-1.10)$ & $1.10(0.90-1.30)$ & $0.260(0.087-0.780)$ & 0.016 \\
\hline $\mathrm{LDL}, \mathrm{mmol} / \mathrm{L}$ & $2.65(2.00-3.20)$ & $2.90(2.20-3.70)$ & $0.721(0.515-1.009)$ & 0.056 \\
\hline HCY, median (IQR), $\mu \mathrm{mol} / \mathrm{L}$ & $15.50(13.00-18.75)$ & $15.00(12.60-19.10)$ & $1.010(0.963-1.060)$ & 0.679 \\
\hline \multicolumn{5}{|l|}{ Clinical characteristics } \\
\hline Injured vessel, $\mathrm{n}(\%)$ & & & $0.846(0.435-1.645)$ & 0.622 \\
\hline Anterior circulation & 30 (78.9) & $174(79.1)$ & & \\
\hline Posterior circulation & $8(21.1)$ & $36(16.4)$ & & \\
\hline Both anterior and posterior cerebral circulation & $0(0)$ & $10(4.5)$ & & \\
\hline CAB, n (\%) ${ }^{11}$ & & & $0.649(0.307-1.371)$ & 0.258 \\
\hline$<50 \%$ stenosis & $12(31.6)$ & $124(56.4)$ & & \\
\hline $50-99 \%$ stenosis & $17(44.7)$ & $61(27.7)$ & & \\
\hline Occlusion & $9(23.7)$ & $35(15.9)$ & & \\
\hline
\end{tabular}


Table I (Continued).

\begin{tabular}{|c|c|c|c|c|}
\hline \multirow[t]{2}{*}{ Parameters } & \multicolumn{2}{|l|}{ Recurrent Stroke } & \multirow[t]{2}{*}{ Unadjusted HR } & \multirow[t]{2}{*}{$P$ value } \\
\hline & Yes $(n=38[14.7 \%])$ & No $(n=220[85.3 \%])$ & & \\
\hline Blood pressure classification, $\mathrm{n}(\%)$ & & & $1.136(0.875-1.475)$ & 0.338 \\
\hline Normal & $10(26.3)$ & $77(35.0)$ & & \\
\hline Grade I & $4(10.5)$ & $17(7.7)$ & & \\
\hline Grade 2 & $10(26.3)$ & $59(26.8)$ & & \\
\hline Grade 3 & $14(36.8)$ & $67(30.5)$ & & \\
\hline Sleep well, n (\%) & $25(65.8)$ & $186(84.5)$ & $0.396(0.202-0.774)$ & 0.007 \\
\hline Admission NIH Stroke Scale, median (IQR) & $3(2-8)$ & $4(2-7)$ & $0.988(0.897-1.089)$ & 0.814 \\
\hline Physical exercise, n (\%) & $21(55.3)$ & $153(69.5)$ & $0.562(0.297-1.066)$ & 0.078 \\
\hline Housework, n (\%) & $7(18.4)$ & 79 (35.9) & $0.422(0.186-0.958)$ & 0.039 \\
\hline
\end{tabular}

Abbreviations: BMI, body mass index; CAB, cerebral atherosclerosis burden; HCY, homocysteine; HDL, high-density lipoprotein; HR, hazard ratio; IHD, ischemic heart disease; IQR, interquartile range; LDL, low-density lipoprotein; TC, total cholesterol; TG, triglyceride.

adjusted HR: 0.474, 95\% CI: 0.238-0.945, $P=0.034$; respectively) as shown in Figures 2-4.

Of note, the variable housework was included in the model since forward elimination Cox proportional hazards regression models were used to calculate adjusted relative risks for RS at 1 year, where the potential confounding factors with $P<0.15$ in the univariate analyses were included. Although housework was not statistically significant, it was a variable in the final equation.

\section{Good Sleep, Active Lifestyle and Smoking Cessation Significantly Decreased the Risk for RS}

The rate of RS during follow-up was significantly lower in patients with good sleep than those with poor sleep (65.8 and 84.5 per 100 patient-years versus, respectively, HR, 0.396; 95\% CI, 0.202-0.774, $P=0.007)$. Patients with daily housework also had a significant reduction in the rate of RS compared with those without by almost $50 \%$ (18.7 per 100 patient-years versus 35.9, respectively, HR, 0.422 ; 95\% CI, 0.186-0.958; $P$ value 0.039 ). When compared with patients without RS, those with RS were more likely to be a smoker (47.4 versus $28.6 \%$, HR, 2.031; 95\% CI, 1.075-3.841; $P=0.029)$. After adjustment for covariates (physical exercise, TC, LDL, hemoglobin $\mathrm{A} 1 \mathrm{C}$, drinking, ischemic heart disease, BMI), smoking was associated with a significantly increased risk of RS for patients after ALVIS (HR, 2.590; 95\% CI, 1.340-5.005; $P=0.005)$. Conversely, the risk of RS was significantly reduced for the patients with good sleep (HR, 0.364;
95\% CI, 0.180-0.739; $P=0.005)$ and patients doing housework (HR, 0.461; 95\% CI, 0.200-1.065; $P=0.070$ ) (Figures 2-4).

\section{Decreased Level of High-Density Lipoprotein (HDL) Was Associated with Increased Risks of RS}

Multivariate Cox regression analyses showed decreased level of high-density lipoprotein (HDL) was significantly associated with increased risks of RS after adjusting for multiple factors (HR, 0.329; 95\% CI, 0.130-0.831; $P=0.019$ ).

\section{Prediction Performance of the Nomogram for RS}

To develop a quantitative model to predict the probability of $\mathrm{RS}$, we constructed a nomogram that integrated both the HDL and lifestyles using the data on the study patients. Multivariate COX hazard regression analyses showed that daily fruit consumption, good sleep, bland diet, housework, smoking, and HDL were independent predictors for RS. A weighted point (from 0 to 100) was given to each risk factor, and the total points from all risk factors for each patient could be used to predict the probability of RS-free for the patient (the lower the total points, the less the risk for $\mathrm{RS}$ ) as shown in Figure 4A and B. For example, a patient with an HDL level of $1.84 \mathrm{mmol} / \mathrm{L}$, daily fruit consumption, no housework, poor sleep, blank diet, and smoking had a total point of 174.5 (45 points for HDL, 0 point for daily fruit consumption, 27 points for housework, 35.5 points for 
A

\begin{tabular}{|c|c|c|c|}
\hline Variables & & HR $(95 \%$ CI) & $p$ value \\
\hline Daily Fruit Consumption & ro- & $0.474(0.238,0.945)$ & 0.034 \\
\hline Housework & - & $0.461(0.200,1.065)$ & 0.070 \\
\hline Good Sleep & $\infty$ & $0.364(0.180,0.739)$ & 0.005 \\
\hline Blank Diet & $\infty$ & $0.365(0.138,0.965)$ & 0.042 \\
\hline Smoking & & $2.590(1.340,5.005)$ & 0.005 \\
\hline HDL & $\infty-1$ & $0.329(0.130,0.831)$ & 0.019 \\
\hline
\end{tabular}

B
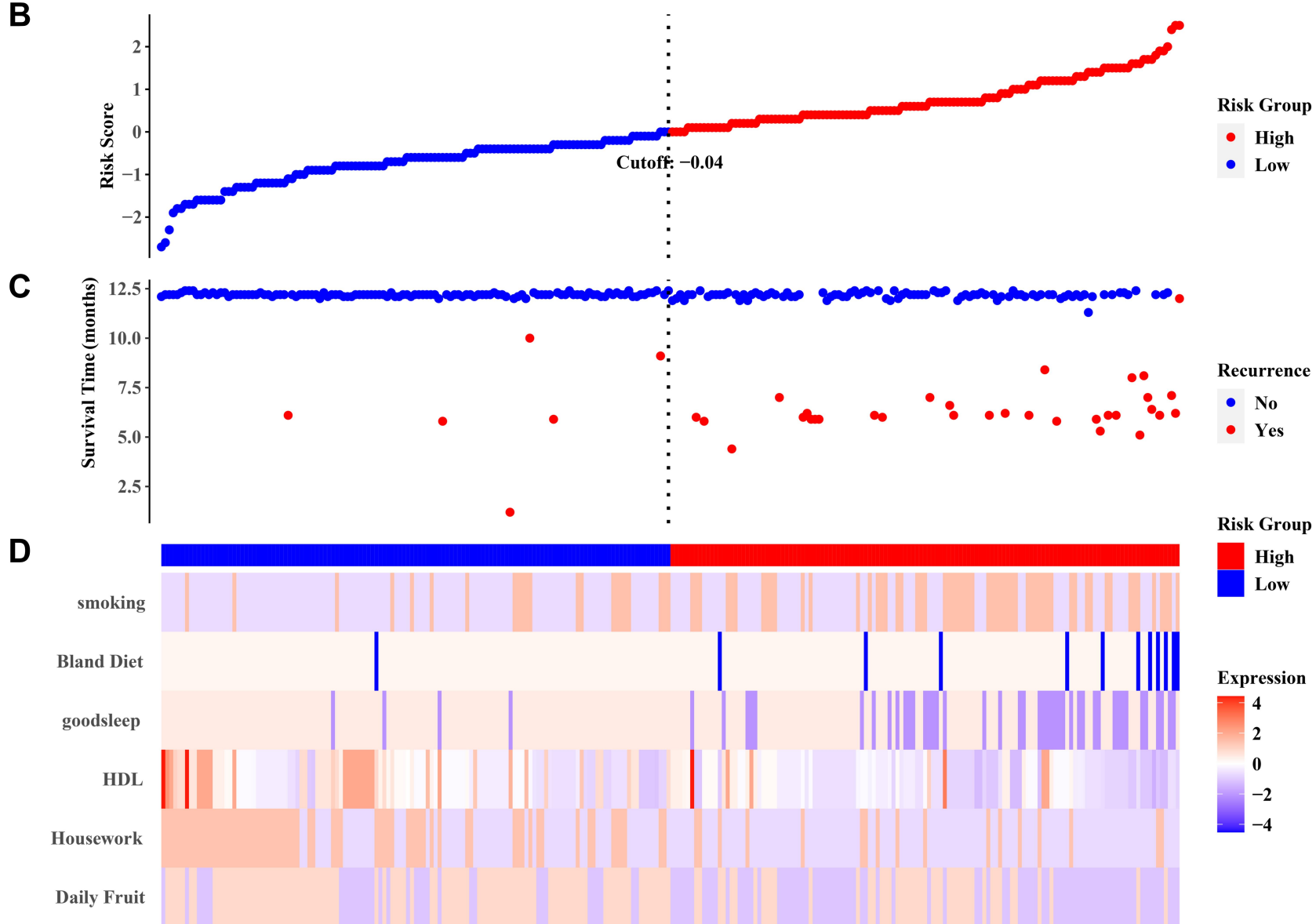

Figure 2 (A). Forest plots of all vascular events by predefined subgroups. Bars represented the relative risk with the $95 \% \mathrm{Cl}$. $\mathrm{P}$ values of the interaction tests were also included. (B-D). Risk scored graph. As expected, high-risk patients had higher stroke recurrence rates and a shorter period of time to develop a stroke recurrence (B and C). In panel (D), the horizontal color bar was colored based on risk groups, corresponding to panel (B). Daily fruit consumption, good sleep, housework, blank diet and $\mathrm{HDL}$ were negatively associated with the risk of stroke recurrence ( $\mathbf{B}$ and $\mathbf{D})$, while smoking significantly increased the risk for stroke recurrence.

Abbreviation: HDL, high-density lipoprotein.

good sleep, 35.5 points for bland diet,31.5 points for smoking). The predicted 1-year RS is $40 \%(1-60.0 \%)$ for this patient. The concordance index of the model was 0.746 (95\% CI $0.675-0.817$ ) with $P$ value of less than 0.001 (Figure 4C).

The patients could be classified into high-risk group and low-risk group according to the patients' linear predictor values as shown in Figure 2B. The cutoff values were about
118 points according to the nomogram (Figure 4A). Based on the total risk score, the corresponding values could be found from the chart that exhibited the predictive values in numerical scale (Linear Predictor).

\section{Discussion}

It is known that patients with large artery cerebrovascular disease have a high risk of early recurrent stroke. Previous 

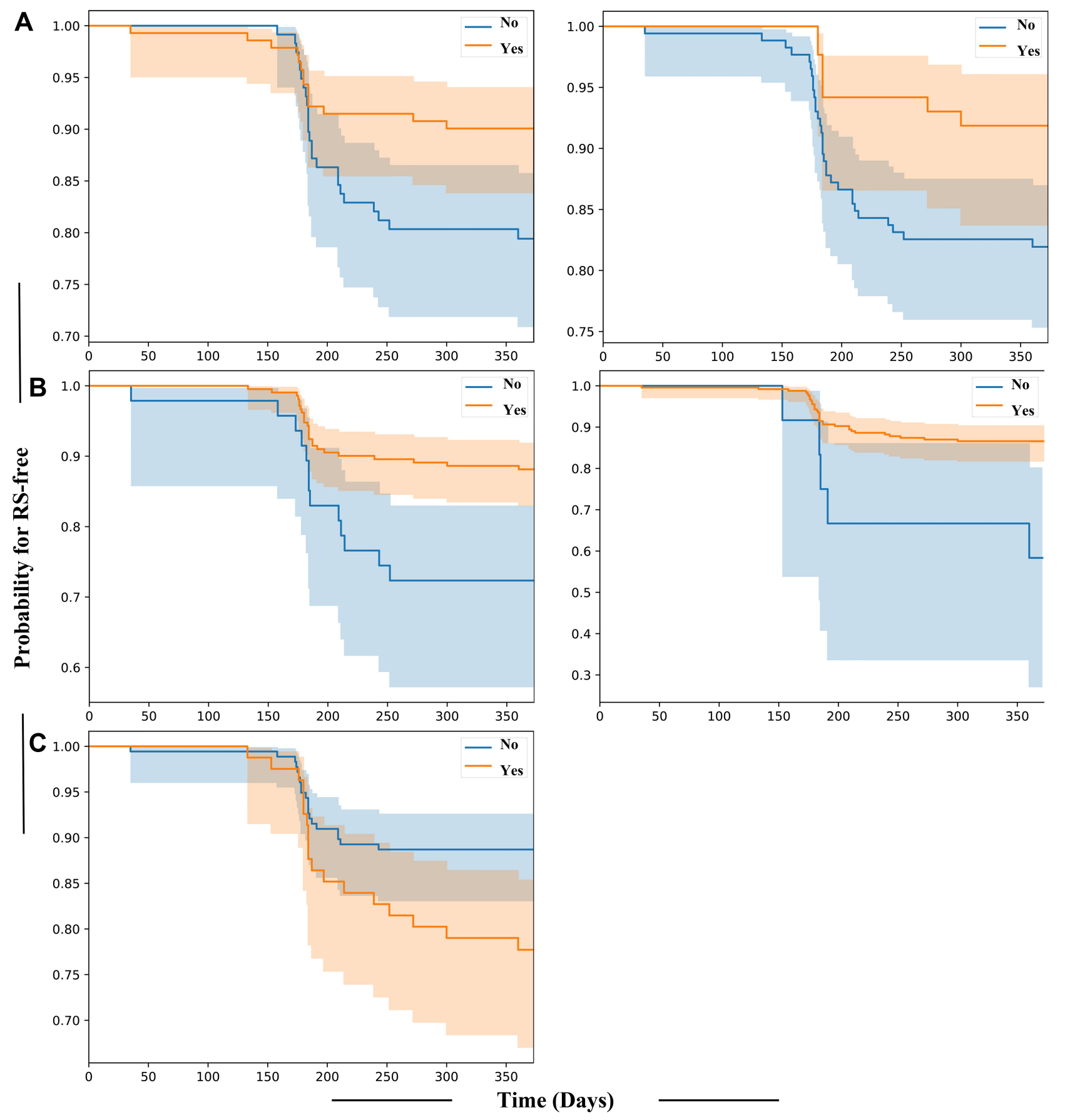

Figure 3 Analyses on RS-free survival for 5 subgroup patients. Kaplan-Meier estimates of cumulative recurrence-free rates of stroke were determined according to daily fruit consumption (A, Left), housework (A, Right), good sleep (B, Left), blank diet (B, Right), and smoking (C) after adjustment for confounding variables. The one-year recurrence-free survival was significantly higher in the non-smoking group of ALVIS patients than that in the smoking group (C).

Abbreviation: RS, recurrent stroke.

studies have suggested that patients with large artery atherosclerosis could have the highest risk of recurrent stroke within 3 months (19.2-19.7\%). ${ }^{12,29}$ The recurrence rate of stroke during one year follow-up was $14.7 \%$ in the present study, which was similar to the findings from these studies. Thus, it is critical to develop an effective strategy to reduce RS risk for patients with large artery stroke like ALVIS. To the best of our knowledge, few studies have reported the association between lifestyle and the risk of RS in Chinese patients with ALVIS. The major findings of the present study were: 1) significantly higher RS risk among stroke patients with ALVIS was associated with 
A

Points

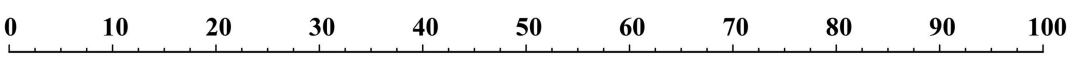

Daily fruit consumption

Housework

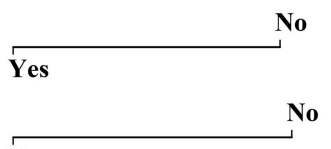

Yes

HDL

Good sleep

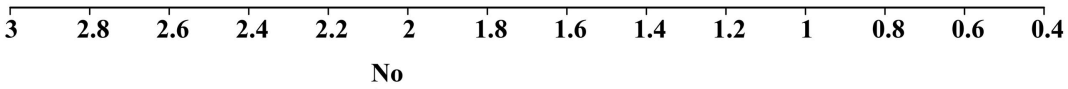

Yes

Bland diet

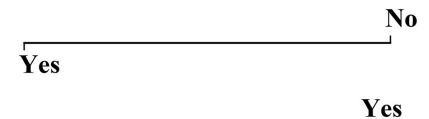

Smoking

No

Total Points

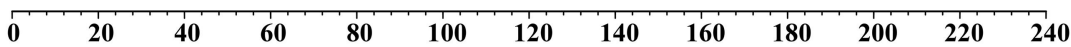

Linear Predictor

$$
\begin{array}{lllllllllllll}
\hline-3 & -2.5 & -2 & -1.5 & -1 & -0.5 & 0 & 0.5 & 1 & 1.5 & 2 & 2.5 & 3
\end{array}
$$

Probability for RS free at 1 year

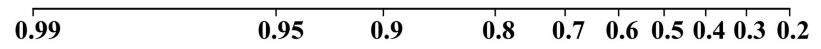

B

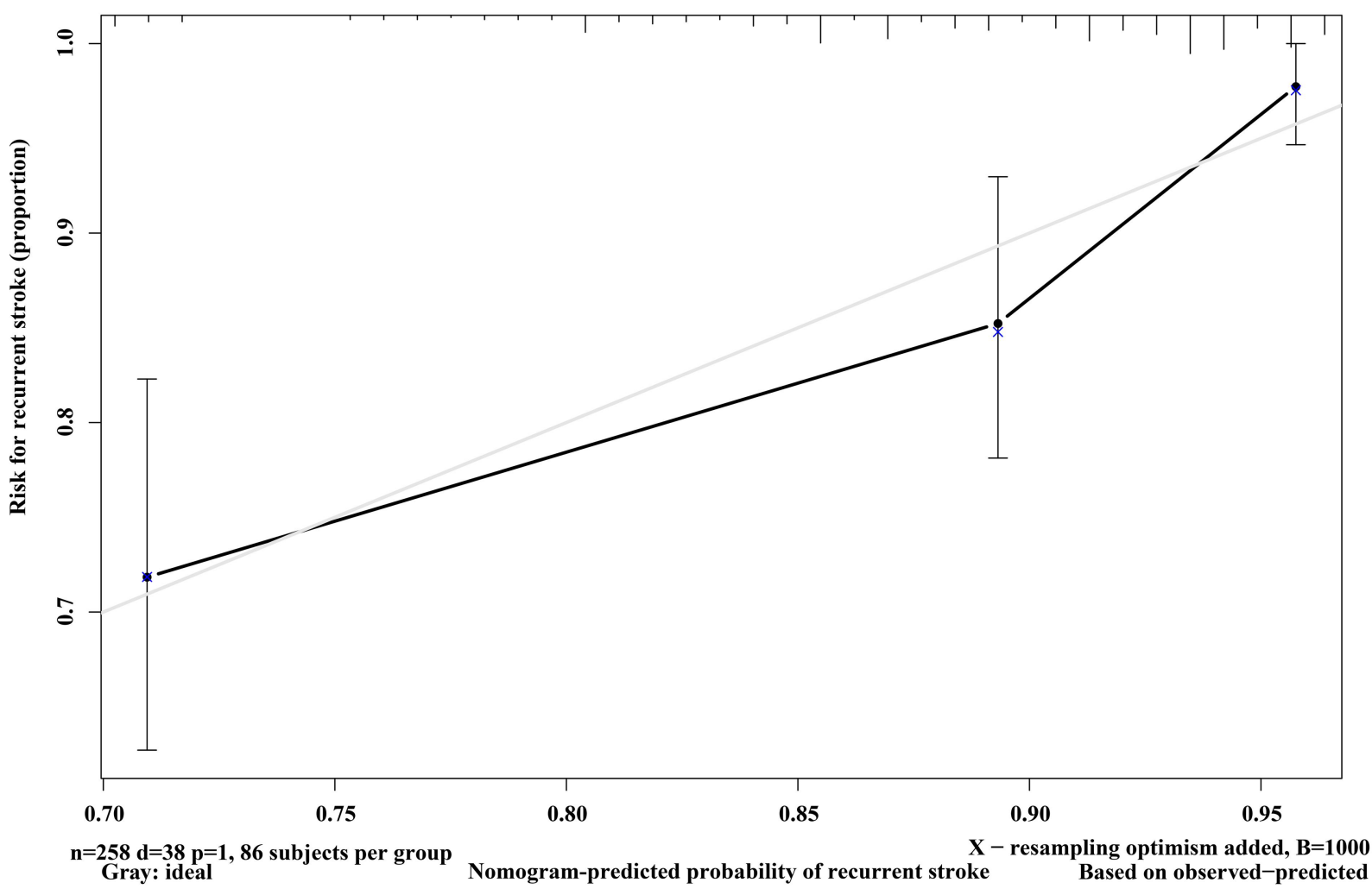

Figure 4 (A) Nomogram for predicting RS. A weighted point (from 0 to 100) was given to each risk factor that could be found in the line marked with "Points" on the top. The total points of all risk factors of each patient could be used to predict the probability of RS-free for the patient by drawing a vertical line from the total points $x$-axis to the probability (the higher the total points, the less the probability for RS-free). The scores for daily fruit consumption, housework, HDL, good sleep, light diet and smoking could be obtained individually by drawing a line from the corresponding value up to the "points line". The "total points" was calculated as the sum of the individual scores of each of the six variables included in the nomogram. (B) Calibration curves of the nomogram to predict RS-free at I year. The actual RS-fee rate for patients was plotted on the y-axis, and nomogram predicted probability for the same patients was plotted on the $\mathrm{x}$-axis. There was a nice match between the actual RS-free rate and the predicted probability.

Abbreviations: HDL, high-density lipoprotein; RS, recurrent stroke. 
unhealthy lifestyle (poor sleep, smoking, salty diet, no daily fruit consumption, or little housework), and 2) admission HDL level independently predicted RS in the patients with ALVIS.

Abundant consumption of fruits, vegetables, folate, fiber, seaweed, and dairy products have been shown to reduce the risk of stroke. ${ }^{30}$ On the other hand, the amount of meat, particularly processed meat, sugary drinks, and high-salt intake are associated with increased risk for stroke. ${ }^{31}$ A previous study revealed that healthy diet with high intake of fruits, vegetables and cereal fiber, low intake of salt and trans fats were associated with reduced stroke risk in men, but not in women. ${ }^{32}$ The end-point events in this study were stroke of all causes (including ischemic, hemorrhagic, and unknown etiology) without adequate power to assess the impact of diet on the risk for stroke related to large arteries. Thus, the present study was focused on the stroke patients with ALVIS, and demonstrated that healthy lifestyle significantly decreased the risk for RS for this patient population. This finding is important since ALVIS represents $20-68 \%$ of all ischemic stroke, and is associated with substantial disability and mortality. ${ }^{33-36}$ Effective prevention of RS for patients with ALVIS could significantly improve the clinical outcome for these patients. The beneficial effect of healthy diet on the recurrence of stroke could be related to high levels of folate, flavonoids (primarily from citrus fruits), and other antioxidants in fruits. ${ }^{37-39}$ Although the exact mechanism for the association between salt intake and stroke is unclear, it might be very likely due to blood pressure regulation, arterial thickness and stiffness as well as platelet reactivity. ${ }^{40}$

One of the interesting findings in the present study was that high quality of sleep was associated with significant risk reduction for RS after ALVIS in Chinese population. It is known that poor sleep is often related to sleep disorders, such as obstructive sleep apnea syndrome $(\mathrm{OSAS})^{41}$ and hypertension, ${ }^{42}$ thus leading to increased risk for stroke and RS. ${ }^{43,44}$ Intermittent hypoxia in OSAS patients may increase the levels of oxidative stress and inflammatory cytokines, leading to endothelial cell dysfunction, arteriosclerosis, and subsequent stroke. $^{45,46}$ Previous studies have shown that both short and long sleep duration are predictors of ischemic stroke. ${ }^{47,48}$ Inadequate sleep could also impair insulin sensitivity, increase sympathetic nervous system and cortisol levels, and alter inflammatory cytokines. ${ }^{49}$ Further studies (both clinical and translational) are needed to investigate the mechanism(s) for the beneficial effect of high-quality sleep on stroke recurrence.
Levels of physical activities are an important measure of functional status especially for the elderly or stroke patients. Since household activities account for a significant portion of total activity in the elderly, and sports and occupational activities usually decrease with age, household activities appear to be the major determinants of non-exercise activity thermogenesis. Indeed, elderly males involved in heavy household chores could consume more calories than those who do not and are strongly associated with better health. ${ }^{50}$ Study also showed that, compared with the reference groups for specific physical activities (up to 1 hour per week walking; 0 hours per week gardening or cycling, and up to 3 hours per week doing housework), women reporting longer durations of these activities tended to have a lower risk of cerebrovascular disease. ${ }^{51}$ Therefore, the post-stroke patients should be encouraged to perform more household chores whenever possible.

The data from the present study also demonstrated that other well-known factors for cardiovascular health and stroke including physical activities (including housework), status of smoking, and serum lipid levels (HDL level) were associated with the risk for RS. ${ }^{51,52}$ A number of studies have confirmed that smoking and passive smoking are important risk factors for AIS. However, there has been little data on the association between smoking and RS. The present study showed that the risk of RS in smokers was 2.6 times that of non-smokers. Smoking could result in a significant increase in heart rate, arterial stiffness, and blood pressure, especially for those with hypertension. ${ }^{53}$ On the other hand, smoking cessation could decrease the risk for RS. ${ }^{54}$ Chronic smokers who quit smoking experienced a significant decrease in blood pressure, heart rate, and arterial stiffness ${ }^{55}$ and increase in HDL. ${ }^{56}$

Dyslipidemia is a major risk factor for RS. Higher serum total cholesterol and LDL were associated with increased risk for RS in Caucasian patients with ALVIS. ${ }^{57}$ However, the impact of HDL on the risk for RS has not been well studied, and some clinical studies have shown that the relationship between HDL and adverse cardiovascular events has been controversial. ${ }^{58}$ In the present study, we observed that HDL was negatively correlated with RS risk in patients with ALVIS after adjustment for the confounding factors. HDL is known to be negatively associated with atherosclerosis. Studies have shown that between 39.4 and $68.0 \%$ ischemic stroke in Chinese population are due to large artery atherosclerosis. ${ }^{34-36} \mathrm{We}$ observed that large artery atherosclerosis comprised $61 \%(258 / 421)$ of all ischemic stroke in the patient cohort in the present study. Thus, it is not surprising that adequate HDL levels are associated with decreased risk 
for RS in patients with ALVIS. The protective nature of HDL against risk for $\mathrm{RS}$ is possibly a combination of multiple beneficial effects HDL on cholesterol transportation for hepatic excretion, ${ }^{59}$ endothelial function, ${ }^{60}$ atherosclerosis, ${ }^{61}$ and oxidative stress and inflammation. ${ }^{62,63}$

Lifestyle is very diverse and complex, and everyone has a different lifestyle with a wide range of variables. Thus, we used a point system to include all the variables to predict the risk for RS for the patients with ALVIS. With this point system, the total score could be used to predict the probability of having a RS after the initial stroke, and to develop an individualized care plan for the patients to minimize the risk of RS. The data from the present study suggested that the point system could predict the risk of RS for the patients with ALVIS. However, a large clinical study is needed to verify the predictive value on risk reduction of $\mathrm{RS}$.

The main strengths of the present study include its prospective design, the detailed lifestyle variables, and the complete follow-up of the subjects in the cohort There were limitations in the present study, including: 1) this was a hospital-based follow-up study, and all patients were recruited from one hospital, thus with limited representation on patient population; 2) The estimates of potentially preventable RS should be viewed as conservative in light of substantial differences in dietary preferences in different regions; 3) The present study was based on selfreported data on lifestyle factors, thus subject to inevitable variations on subjective perception. For example, the definition of a bland diet was also based on self-report from patients and caregivers without quantitative criteria like a detailed calculation of daily salt intake. The use of prospectively collected data and cumulative averages based on repeated evaluations would reduce the impact of random measurement errors. 4) We only analyzed the NIHSS score at admission and mRS during follow-up, but not the NIHSS score at discharge with no data on the difference between the NIHSS score at admission and discharge, which needs further exploration. 5) A multicenter validation study is needed to confirm the predictive values of the nomogram. And 6) the patients in the present study were predominantly males. Future studies are needed to determine if there is a sex difference in the effect of lifestyle on RS risk for patients with ALVIS.

\section{Conclusion}

The present study demonstrated healthy lifestyle, including healthy diet, good sleep, physical activities, and smoking cessation, plays an important role in preventing RS in patients with ALVIS. The nomogram based on lifestyle could be used to predict the probability of RS for patients and to develop an individualized care plan to achieve optimal outcome for patients with ALVIS by minimizing the risk of $\mathrm{RS}$.

\section{Statement of Ethics}

The study protocol was approved by the Ethical Committee of Guangdong Second Provincial General Hospital (approval number: 20,120,812-GD2H).

\section{Author Contributions}

All authors made substantial contributions to conception and design, acquisition of data, or analysis and interpretation of data; took part in drafting the article or revising it critically for important intellectual content; agreed to submit to the current journal; gave final approval of the version to be published; and agree to be accountable for all aspects of the work.

\section{Funding}

This work was partially supported by the Science and Technology Program of Guangzhou, China (201707010436), Medical Scientific Research Foundation of Guangdong Province, China (B2019068; C2019056) and Administration of Traditional Chinese Medicine of Guangdong Province, China (20181012). The funders had no role in the design and conduct of the study, in the collection, analysis, and interpretation of the data, and in the preparation, review, or approval of the manuscript.

\section{Disclosure}

The authors report no conflicts of interest in this work.

\section{References}

1. Virani SS, Alonso A, Benjamin EJ, et al. Heart disease and stroke statistics-2020 update: a report from the american heart association. Circulation. 2020;141:e139-e596.

2. Hankey GJ. Secondary stroke prevention. Lancet Neurol. 2014;13:178-194. doi:10.1016/S1474-4422(13)70255-2

3. Liu J, Zhu Y, Wu Y, Liu Y, Teng Z, Hao Y. Association of carotid atherosclerosis and recurrent cerebral infarction in the Chinese population: a meta-analysis. Neuropsychiatr Dis Treat. 2017;13:527-533. doi:10.2147/NDT.S124386

4. Coull AJ, Rothwell PM. Underestimation of the early risk of recurrent stroke: evidence of the need for a standard definition. Stroke. 2004;35:1925-1929. doi:10.1161/01.STR.0000133129.58126.67

5. Chimowitz MI, Lynn MJ, Derdeyn CP, et al. Stenting versus aggressive medical therapy for intracranial arterial stenosis. $N$ Engl J Med. 2011;365:993-1003. doi:10.1056/NEJMoa1105335 
6. Banerjee C, Chimowitz MI. Stroke caused by atherosclerosis of the major intracranial arteries. Circ Res. 2017;120:502-513. doi:10.1161/ CIRCRESAHA.116.308441

7. O’Donnell MJ, Chin SL, Rangarajan S, et al. Global and regional effects of potentially modifiable risk factors associated with acute stroke in 32 countries (INTERSTROKE): a case-control study. Lancet. 2016;388:761-775. doi:10.1016/S0140-6736(16)30506-2

8. Johnson CO, Nguyen M, Roth GA. Global, regional, and national burden of stroke, 1990-2016: a systematic analysis for the global burden of disease study 2016. Lancet Neurol. 2019;18:439-458. doi:10.1016/S1474-4422(19)30034-1

9. Turan TN, Al Kasab S, Nizam A, et al. Relationship between risk factor control and compliance with a lifestyle modification program in the stenting aggressive medical management for prevention of recurrent stroke in intracranial stenosis trial. J Stroke Cerebrovasc Dis. 2018;27:801-805. doi:10.1016/j.jstrokecerebrovasdis.2017.10.017

10. Diener HC, Hankey GJ. Primary and secondary prevention of ischemic stroke and cerebral hemorrhage: JACC focus seminar. $J$ Am Coll Cardiol. 2020;75:1804-1818. doi:10.1016/j.jacc.2019.12.072

11. Amarenco P, Bogousslavsky J, Caplan LR, Donnan GA, Hennerici MG. Classification of stroke subtypes. Cerebrovasc Dis. 2009;27:493-501. doi:10.1159/000210432

12. Lovett JK, Coull AJ, Rothwell PM. Early risk of recurrence by subtype of ischemic stroke in population-based incidence studies. Neurology. 2004;62:569-573. doi:10.1212/01.WNL.0000110311.09970.83

13. Kim JT, Yoo SH, Kwon JH, Kwon SU, Kim JS. Subtyping of ischemic stroke based on vascular imaging: analysis of 1167 acute, consecutive patients. J Clin Neurol. 2006;2:225-230. doi:10.3988/ jen.2006.2.4.225

14. Kim D, Lee SH, Joon Kim B, et al. Secondary prevention by stroke subtype: A nationwide follow-up study in 46108 patients after acute ischaemic stroke. Eur Heart J. 2013;34:2760-2767. doi:10.1093/eurheartj/eht185

15. Liu W, Wang K, Han Y, Liang JY, Li YH, Xing BC. Nomogram predicted disease free survival for colorectal liver metastasis patients with preoperative chemotherapy followed by hepatic resection. Eur J Surg Oncol. 2019;45:2070-2077. doi:10.1016/j.ejso.2019.06.033

16. Wang XH, Liao B, Hu WJ, et al. Novel models predict postsurgical recurrence and overall survival for patients with hepatitis $b$ virusrelated solitary hepatocellular carcinoma $\leq 10 \mathrm{~cm}$ and without portal venous tumor thrombus. Oncologist. 2020;25:e1552-61. doi:10.1634/ theoncologist.2019-0766

17. Chung JW, Park SH, Kim N, et al. Trial of ORG 10172 in Acute Stroke Treatment (TOAST) classification and vascular territory of ischemic stroke lesions diagnosed by diffusion-weighted imaging. $J$ Am Heart Assoc. 2014;11:e01119.

18. Qureshi AI, Kirmani JF, Sayed MA, et al. Time to hospital arrival, use of thrombolytics, and in-hospital outcomes in ischemic stroke. Neurology. 2005;64:2115-2120. doi:10.1212/01. WNL.0000165951.03373.25

19. Turan TN, Hertzberg V, Weiss P, et al. Clinical characteristics of patients with early hospital arrival after stroke symptom onset. $J$ Stroke Cerebrovasc Dis. 2005;14:272-277. doi:10.1016/j. jstrokecerebrovasdis.2005.07.002

20. Leon-Jimenez C, Ruiz-Sandoval JL, Chiquete E, et al. Hospital arrival time and functional outcome after acute ischaemic stroke: results from the premier study. Neurologia. 2014;29:200-209. doi:10.1016/j.nrl.2013.05.003

21. Silvestrelli G, Parnetti L, Paciaroni M, et al. Early admission to stroke unit influences clinical outcome. Eur $J$ Neurol. 2006;13:250-255. doi:10.1111/j.1468-1331.2006.01187.x

22. Weimar C, Ziegler A, Konig IR, Diener HC. Predicting functional outcome and survival after acute ischemic stroke. J Neurol. 2002;249:888-895. doi:10.1007/s00415-002-0755-8
23. Appelros P, Nydevik I, Viitanen M. Poor outcome after first-ever stroke: predictors for death, dependency, and recurrent stroke within the first year. Stroke. 2003;34:122-126. doi:10.1161/01. STR.0000047852.05842.3C

24. Huang ZX, Lin XL, Lu HK, Liang XY, Fan LJ, Liu XT. Lifestyles correlate with stroke recurrence in Chinese inpatients with first-ever acute ischemic stroke. J Neurol. 2019;266:1194-1202. doi:10.1007/ s00415-019-09249-5

25. Li Y, Schoufour J, Wang DD, et al. Healthy lifestyle and life expectancy free of cancer, cardiovascular disease, and type 2 diabetes: prospective cohort study. $B M J$. 2020;368:16669. doi:10.1136/bmj. 16669

26. Sobieraj A, Fabin K, Wilczyński K, Krysta K. Occurrence of sleep abnormalities among people with mental disorders-questionnaire study. Psychiatr Danub. 2013;25:S203-6.

27. Platt JM, Bates LM, Jager J, McLaughlin KA, Keyes KM. Changes in the depression gender gap from 1992 to 2014: cohort effects and mediation by gendered social position. Soc Sci Med. 2020;258:113088. doi:10.1016/j.socscimed.2020.113088

28. Jansen SC, Abaraogu UO, Lauret GJ, Fakhry F, Fokkenrood HJ, Teijink JA. Modes of exercise training for intermittent claudication. Cochrane Database Syst Rev. 2020;8:CD009638. doi:10.1002/ 14651858.CD009638.pub3

29. Shin DH, Lee PH, Bang OY. Mechanisms of recurrence in subtypes of ischemic stroke: A hospital-based follow-up study. Arch Neurol. 2005;62:1232-1237. doi:10.1001/archneur.62.8.1232

30. Larsson SC, Wallin A, Wolk A. Dietary approaches to stop hypertension diet and incidence of stroke: results from 2 prospective cohorts. Stroke. 2016;47:986-990. doi:10.1161/STROKEAHA.116.012675

31. Hankey GJ. The role of nutrition in the risk and burden of stroke: an update of the evidence. Stroke. 2017;48:3168-3174. doi:10.1161/ STROKEAHA.117.016993

32. Chiuve SE, Rexrode KM, Spiegelman D, Logroscino G, Manson JE, Rimm EB. Primary prevention of stroke by healthy lifestyle. Circulation. 2008;118(9):947-954. doi:10.1161/ CIRCULATIONAHA.108.781062

33. Ornello R, Degan D, Tiseo C, et al. Distribution and temporal trends from 1993 to 2015 of ischemic stroke subtypes: a systematic review and meta-analysis. Stroke. 2018;49:814-819. doi:10.1161/ STROKEAHA.117.020031

34. Hong YH, Zhou LX, Yao M, et al. Lesion topography and its correlation with etiology in medullary infarction: analysis from a multi-center stroke study in china. Front Neurol. 2018;9:813. doi:10.3389/fneur.2018.00813

35. Yang C, Zhang J, Liu C, Xing Y. Comparison of the risk factors of hemorrhagic transformation between large artery atherosclerosis stroke and cardioembolism after intravenous thrombolysis. Clin Neurol Neurosurg. 2020;196:106032. doi:10.1016/j. clineuro.2020.106032

36. Tang M, Yao M, Zhu Y, Peng B, Zhou L, Ni J. Sex differences of ischemic stroke in young adults-a single-center chinese cohort study. J Stroke Cerebrovasc Dis. 2020;29:105087. doi:10.1016/j. jstrokecerebrovasdis.2020.105087

37. Hankey GJ. Vitamin supplementation and stroke prevention. Stroke. 2012;43:2814-2818. doi:10.1161/STROKEAHA.111.639930

38. Xu YJ, Tappia PS, Neki NS, Dhalla NS. Prevention of diabetes-induced cardiovascular complications upon treatment with antioxidants. Heart Fail Rev. 2014;19:113-121. doi:10.1007/s10741013-9379-6

39. Hankey GJ. B vitamins for stroke prevention. Stroke Vasc Neurol. 2018;3:51-58. doi:10.1136/svn-2018-000156

40. de Wardener HE, MacGregor GA. Harmful effects of dietary salt in addition to hypertension. J Hum Hypertens. 2002;16:213-223. doi:10.1038/sj.jhh.1001374 
41. Yaggi HK, Concato J, Kernan WN, Lichtman JH, Brass LM, Mohsenin V. Obstructive sleep apnea as a risk factor for stroke and death. $N$ Engl $J$ Med. 2005;353:2034-2041. doi:10.1056/ NEJMoa043104

42. Brown DL, Shafie-Khorassani F, Kim S, et al. Sleep-disordered breathing is associated with recurrent ischemic stroke. Stroke. 2019;50:571-576. doi:10.1161/STROKEAHA.118.023807

43. Williams B, Mancia G, Spiering W, et al. 2018 ESC/ESH guidelines for the management of arterial hypertension: the task force for the management of arterial hypertension of the european society of cardiology and the european society of hypertension: the task force for the management of arterial hypertension of the european society of cardiology and the european society of hypertension. J Hypertens. 2018;36:1953-2041. doi:10.1097/HJH.0000000000001940

44. Kohler M, Stradling JR. Mechanisms of vascular damage in obstructive sleep apnea. Nat Rev Cardiol. 2010;7:677-685. doi:10.1038/ nrcardio. 2010.145

45. Rubies C, Dantas A-P, Batlle M, et al. Aortic remodelling induced by obstructive apneas is normalized with mesenchymal stem cells infusion. Sci Rep. 2019;9(1):11443. doi:10.1038/s41598-019-47813-1

46. Arnaud C, Bouyon S, Recoquillon S. et al. Nonmuscle myosin light chain kinase: a key player in intermittent hypoxia-induced vascular alterations. $J$ Am Heart Assoc;2018. 7. doi: 10.1161/ JAHA.117.007893

47. Itani O, Jike M, Watanabe N, Kaneita Y. Short sleep duration and health outcomes: a systematic review, meta-analysis, and meta-regression. Sleep Med. 2017;32:246-256. doi:10.1016/j. sleep.2016.08.006

48. Jike M, Itani O, Watanabe N, Buysse DJ, Kaneita Y. Long sleep duration and health outcomes: A systematic review, meta-analysis and meta-regression. Sleep Med Rev. 2018;39:25-36. doi:10.1016/j. smrv.2017.06.011

49. Tobaldini E, Fiorelli EM, Solbiati M, Costantino G, Nobili L, Montano N. Short sleep duration and cardiometabolic risk: from pathophysiology to clinical evidence. Nat Rev Cardiol. 2019;16:213-224. doi:10.1038/s41569-018-0109-6

50. Yu R, Leung J, Woo J. Housework reduces all-cause and cancer mortality in chinese men. PLoS One. 2013;8:e61529. doi:10.1371/ journal.pone.0061529

51. Armstrong ME, Green J, Reeves GK, Beral V, Cairns BJ. Frequent physical activity may not reduce vascular disease risk as much as moderate activity: large prospective study of women in the united kingdom. Circulation. 2015;131:721-729. doi:10.1161/CIRCULA TIONAHA.114.010296
52. Sullivan GM, Feinn R. Using effect size-or why the p value is not enough. J Grad Med Educ. 2012;4:279-282. doi:10.4300/JGMED-12-00156.1

53. Bhatnagar A, Maziak W, Eissenberg T, et al. Water pipe (hookah) smoking and cardiovascular disease risk: a scientific statement from the american heart association. Circulation. 2019;139:e917-e936. doi:10.1161/CIR.0000000000000671

54. Epstein KA, Viscoli CM, Spence JD, et al. Smoking cessation and outcome after ischemic stroke or TIA. Neurology. 2017;89:1723-1729. doi:10.1212/WNL.0000000000004524

55. Jatoi NA, Jerrard-Dunne P, Feely J, Mahmud A. Impact of smoking and smoking cessation on arterial stiffness and aortic wave reflection in hypertension. Hypertension. 2007;49:981-985. doi:10.1161/ HYPERTENSIONAHA.107.087338

56. März W, Kleber ME, Scharnagl H, et al. HDL cholesterol: reappraisal of its clinical relevance. Clin Res Cardiol. 2019;139(9):663-675. doi:10.1007/s00392-017-1106-1

57. Amarenco P, Benavente O, Goldstein LB, et al. Results of the stroke prevention by aggressive reduction in cholesterol levels (SPARCL) trial by stroke subtypes. Stroke. 2009;40:1405-1409. doi:10.1161/ STROKEAHA.108.534107

58. Tardif JC, Ballantyne CM, Barter P, et al. Effects of the high-density lipoprotein mimetic agent CER-001 on coronary atherosclerosis in patients with acute coronary syndromes: a randomized trial. Eur Heart J. 2014;35:3277-3286. doi:10.1093/eurheartj/ehu171

59. Glomset JA, Janssen ET, Kennedy R, Dobbins J. Role of plasma lecithin: cholesterol acyltransferase in the metabolism of high density lipoproteins. J Lipid Res. 1966;7:638-648. doi:10.1016/S00222275(20)39245-2

60. Bisoendial RJ, Hovingh GK, Levels JH, et al. Restoration of endothelial function by increasing high-density lipoprotein in subjects with isolated low high-density lipoprotein. Circulation. 2003;107:2944-2948. doi:10.1161/01.CIR.0000070934.69310.1A

61. Rye KA, Barter PJ. Cardioprotective functions of HDLs. J Lipid Res. 2014;55:168-179. doi:10.1194/jlr.R039297

62. Parthasarathy S, Barnett J, Fong LG. High-density lipoprotein inhibits the oxidative modification of low-density lipoprotein. Biochim Biophys Acta. 1990;1044:275-283. doi:10.1016/0005-2760(90) 90314-N

63. Sang $\mathrm{H}$, Yao S, Zhang L, et al. Walk-run training improves the anti-inflammation properties of high-density lipoprotein in patients with metabolic syndrome. $J$ Clin Endocrinol Metab. 2015;100:870-879. doi:10.1210/jc.2014-2979
Risk Management and Healthcare Policy

\section{Publish your work in this journal}

Risk Management and Healthcare Policy is an international, peerreviewed, open access journal focusing on all aspects of public health, policy, and preventative measures to promote good health and improve morbidity and mortality in the population. The journal welcomes submitted papers covering original research, basic science, clinical \& epidemiological studies, reviews and evaluations, guidelines, expert opinion and commentary, case reports and extended reports. The manuscript management system is completely online and includes a very quick and fair peer-review system, which is all easy to use. Visit http://www.dovepress.com/testimonials.php to read real quotes from published authors. 